Archive for

Organic Chemistry

Arkivoc 2018, part vii, 0-0

\title{
Synthesis and properties of polymer chemosensors based on 1-vinylimidazole
}

\author{
Vladimir A. Bren, ${ }^{\text {a } I v a n ~ E . ~ T o l p y g i n, ~}{ }^{\text {a }}$ Yurii V. Revinskii, ${ }^{b}$ Karina S. Tikhomirova, ${ }^{a}$ \\ Alexander D. Dubonosov, ${ }^{* b}$ and Vladimir I. Minkin ${ }^{a, b}$ \\ a Institute of Physical and Organic Chemistry, Southern Federal University, 194/2, Stachka Av., \\ 344090 Rostov on Don, Russian Federation \\ ${ }^{b}$ Southern Scientific Center of Russian Academy of Sciences, 41, Chekhov Pr., \\ 344006 Rostov on Don, Russian Federation \\ Email: aled@ipoc.sfedu.ru
}

Received 05-18-2018

Accepted 08-10-2018

Published on line $08-25-2018$

\section{Abstract}

Derivatives of poly(1-vinylimidazolium chloride) possessing chemosensor properties to cations and anions were synthesized by quaternization of poly-(1-vinylimidazole) (PVI) $\left(M_{w} \sim 63000\right)$ by alkylating compounds containing anthracene fluorophore. Poly(\{3-[2-(anthracen-9-ylmethyl)amino]ethyl\}-1-vinylimidazolium chloride) demonstrates selectivity with more than a 20 fold increase in relative fluorescence intensity $I / I_{0}$ in the presence of $\mathrm{Zn}^{2+}$ cations. Poly(\{3-[2-(anthracen-9-ylmethyl)(3,5-dichlorophenyl)amino]-2-oxoethyl\}-1-vinylimidazolium chloride) acts as a chemosensor for $\mathrm{AcO}^{-}$, changing the color of its DMSO solution from pale yellow to bright orange.

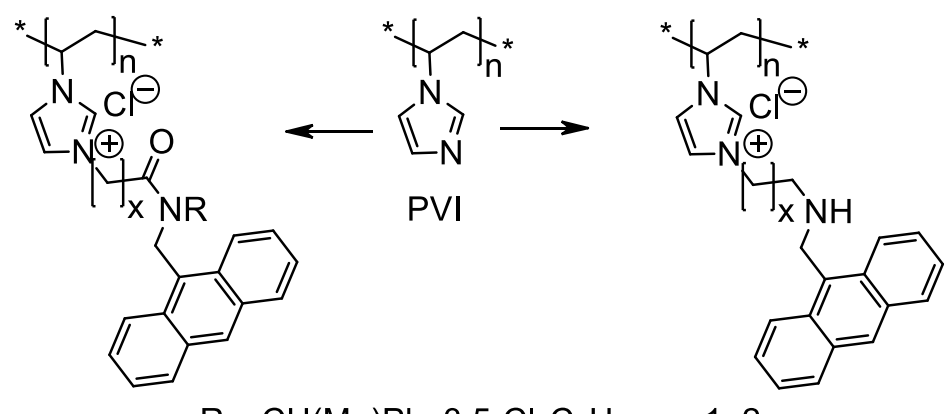

Keywords: Poly(1-vinylimidazole), anthracene, quaternization, fluorescence, chemosensor 


\section{Introduction}

Chemosensor materials are widely exploited for the rapid qualitative and quantitative analysis of ions and molecules in organic and inorganic samples. ${ }^{1-7}$ They are in demand in various fields of science and technology for environmental monitoring, determining food quality, and purity of medical drugs. ${ }^{8-11}$ Polymer systems containing chelating fragments are used not only as chemosensors, but also for production of ionic liquids, OLED materials and liquid crystals. ${ }^{12-17}$ Compared with organic low-molecular chemosensors, polymer-based chemosensors potentially have a number of advantages: enhanced emission signals, increased binding efficiency and recognition selectivity, and simple conversion into devices. ${ }^{18-21}$ Polymers bearing pendant heterocyclic moieties containing chemically active centers are promising macromolecular frameworks for the creation of chemosensor materials.

Herein, we have chosen poly-(1-vinylimidazole) (PVI) containing highly basic nitrogen atoms of the pyridine rings prone to quaternization reactions. Substituted aminoalkyl chlorides and chlorocarboxylic acid amides with anthracene substituent were used as alkylating agents. The anthryl fluorophore is a simple and efficient signaling fragment of chemosensors for determination of cations and anions. ${ }^{22-24}$ Thus, the goals of this study were the synthesis of polymers containing functionalized imidazolium groups and investigation of their interaction with ions.

\section{Results and Discussion}

Alkylating agents 2-7 possessing the anthracene fluorophore were synthesized according to Scheme 1.

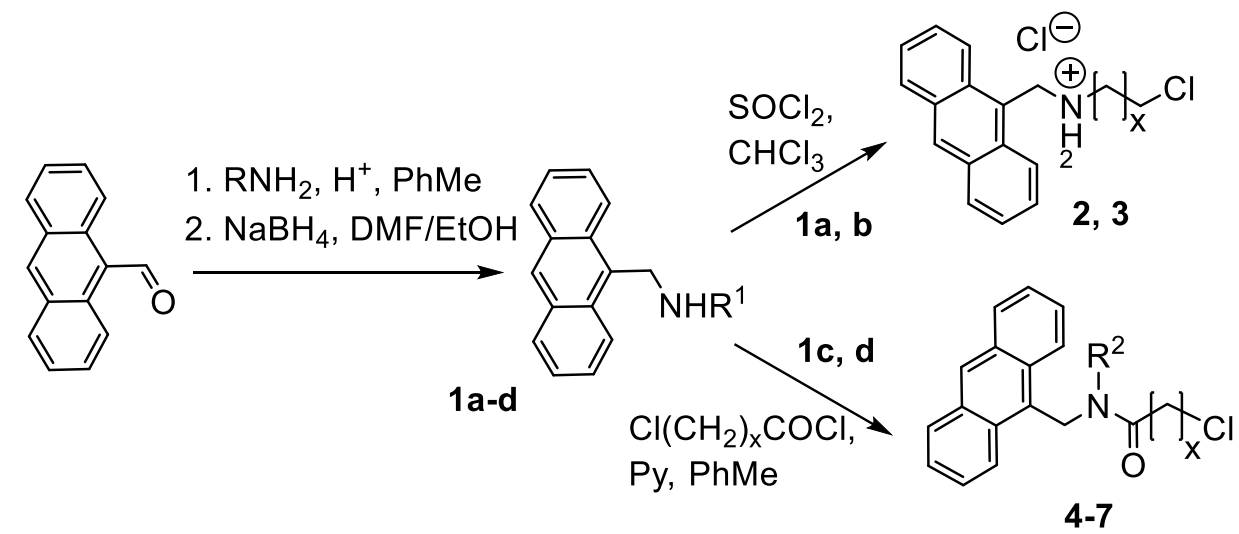

1, $\mathrm{R}^{1}=\left(\mathrm{CH}_{2}\right)_{2} \mathrm{OH}(\mathbf{a}) ;\left(\mathrm{CH}_{2}\right)_{3} \mathrm{OH}(\mathbf{b}) ; \mathrm{CH}(\mathrm{Me}) \mathrm{Ph}(\mathbf{c}) ; 3,5-\mathrm{Cl}_{2} \mathrm{C}_{6} \mathrm{H}_{3}(\mathbf{d}) ;$
$\mathbf{4}, \mathbf{6}, \mathrm{R}^{2}=\mathrm{CH}(\mathrm{Me}) \mathrm{Ph} ; \mathbf{5}, \mathbf{7}, \mathrm{R}^{2}=3,5-\mathrm{Cl}_{2} \mathrm{C}_{6} \mathrm{H}_{3} ; \mathbf{2}, \mathbf{4}, \mathbf{5}, \mathrm{x}=1 ; \mathbf{3}, \mathbf{6}, \mathbf{7}, \mathrm{x}=\mathbf{2}$

Scheme 1. Synthesis of aminoalkyl chlorides and chlorocarboxylic acid amides 2-7.

Compounds 1a-d were prepared by condensation of the corresponding amines with anthracene-9carbaldehyde and the subsequent reduction of the resulting azomethines by $\mathrm{NaBH}_{4}$. The reaction was monitored by the appearance in the ${ }^{1} \mathrm{H}$ NMR spectra of characteristic proton signals of the $\mathrm{CH}_{2}$ groups linking the anthracene fragment and $\mathrm{NH}$ groups, as well as by the disappearance of the methine proton signals of the intermediate azomethines. Amino alcohols $\mathbf{1 a}$ and $\mathbf{1 b}$ were chlorinated by $\mathrm{SOCl}_{2}$ to form chloroalkyl amines $\mathbf{2}$ and $\mathbf{3}$, respectively. Interaction of amines $\mathbf{1 c}$ and $\mathbf{1 d}$ with 2-chloroacetyl and 3-chloropropanoyl chlorides gave 
rise to the corresponding chloroamides $\mathbf{4 - 7}$. Acylation of amines $\mathbf{1} \mathbf{c}$ and $\mathbf{1} \mathbf{d}$ is accompanied by the appearance of additional proton signals of $\mathrm{CH}_{2}$ group detected by the ${ }^{1} \mathrm{H}$ NMR spectra and characteristic carbonyl absorption bands in the IR spectra.

The macromolecular structures 8-13 were synthesized by quaternization of poly-(1-vinylimidazole) with an excess of the obtained alkylating agents 2-7 (Scheme 2). According to the intensity ratios for the proton signals of the $\left(\mathrm{CHCH}_{2}\right)_{n}$ fragments of the polymer chain and the $\left(\mathrm{CH}_{2}\right)_{n}$ groups connected with the anthryl fluorophores, as registered by ${ }^{1} \mathrm{H}$ NMR, quaternization of the imidazole moieties was a fully exhaustive process.

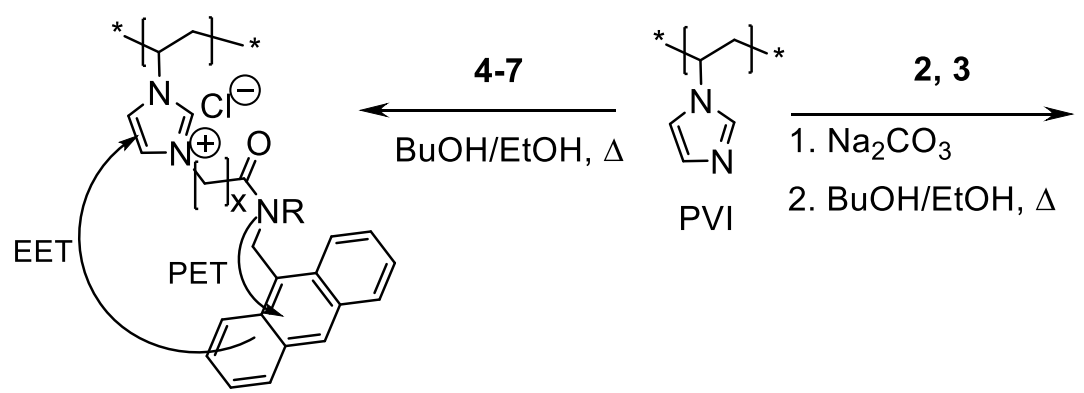

10-13
10, $12 \mathrm{R}=\mathrm{CH}(\mathrm{Me}) \mathrm{Ph} ; 11,13 \mathrm{R}=3,5-\mathrm{Cl}_{2} \mathrm{C}_{6} \mathrm{H}_{3}$;

$8,10,11, x=1 ; 9,12,13, x=2$

$\mathrm{n}$

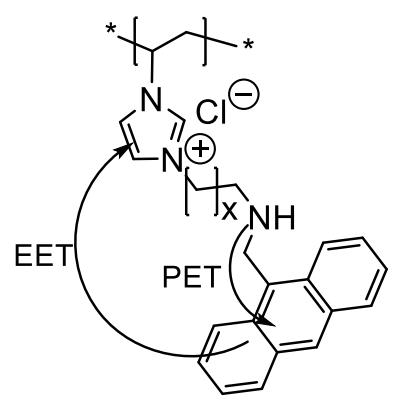

8,9

$\mathrm{n}$

Scheme 2. Synthesis of poly-(1-vinylimidazolium) derivatives 8-13.

The electronic absorption spectra of polymers 8-13 measured in DMSO solution are represented by the structured bands in the region of $350-390 \mathrm{~nm}$. When excited with the light $\lambda_{\text {exc }}=370 \mathrm{~nm}$ the solutions display low-intensity anthracene-type fluorescence (three individual maxima at 400-470 nm and a shoulder at $\sim 480$ $\mathrm{nm}$ ). The fluorescence quantum yields for amines 8 and 9 were 0.008 and 0.007 , respectively, and for amides 10-13 0.005. Previously, we have found that chemosensors containing a 9-aminomethylanthracene fragment, manifest the PET effect (Photoinduced Electron Transfer) in the excited state due to the effective interaction of the unshared electron pair of the nitrogen atom with the anthracene moiety. As a rule, the emission intensity in such systems did not exceed the value of $\varphi 0.001 .23,24$ The described polymer polymers 813 possess noticeably more intense emission. Tentatively, we attributed this to two competing processes acting in different directions - PET effect and EET effect caused by the electron density transfer from the donor (anthryl) part of the molecule to the acceptor (imidazolium) fragment occurring in the excited state (Scheme 2). ${ }^{25,26}$

Investigation the interaction of polymers 8-13 in DMSO with $\mathrm{Zn}^{2+}, \mathrm{Cd}^{2+}, \mathrm{Cu}^{2+}, \mathrm{Ni}^{2+}, \mathrm{Pb}^{2+}, \mathrm{Hg}^{2+}$ cations (in the form of perchlorates) showed that for aminoalkyl polymers $\mathbf{8}$ and $\mathbf{9}$, the most significant changes in the fluorescence intensity were caused by zinc (II) cations, which produce a 21 and 14 fold enhancement of the emission of solutions of $\mathbf{8}$ and $\mathbf{9}$, respectively (Table 1, Figure 1). It was shown earlier that even one NH group is enough for effective binding of $d$-metal ions, inhibition of the PET effect and a significant increase in the emission intensity. ${ }^{23}$ The result was also supported by DFT calculations that predicted additional cation coordination with anthracene $\pi$ system and the highest energy of complex formation specifically for $\mathrm{Zn}^{2+} \cdot{ }^{27}$ 
Table 1. Relative changes in fluorescence intensities of polymers 8-13 (c 5.0.10-6 M) in DMSO upon addition of cations (in the form of perchlorates) $\left(c 2.5 \cdot 10^{-5} \mathrm{M}\right.$ )

\begin{tabular}{ccccccc}
\hline \multirow{2}{*}{ Polymer } & \multicolumn{6}{c}{ Relative change in fluorescence intensity, $\mathrm{I} / \mathrm{I}_{0}$} \\
\cline { 2 - 7 } & $\mathrm{Zn}^{2+}$ & $\mathrm{Cd}^{2+}$ & $\mathrm{Cu}^{2+}$ & $\mathrm{Ni}^{2+}$ & $\mathrm{Pb}^{2+}$ & $\mathrm{Hg}^{2+}$ \\
\hline $\mathbf{8}$ & 21.0 & 4.0 & 0.6 & 1.0 & 1.0 & 4.0 \\
$\mathbf{9}$ & 14.0 & 7.0 & 0.8 & 0.8 & 1.0 & 3.0 \\
10 & 1.6 & 1.1 & 0.5 & 0.2 & 0.8 & 0.5 \\
$\mathbf{1 1}$ & 2.2 & 1.6 & 0.8 & 0.6 & 1.1 & 1.2 \\
12 & 1.2 & 1.0 & 0.8 & 0.7 & 0.9 & 1.0 \\
13 & 1.0 & 1.1 & 0.7 & 0.5 & 1.0 & 0.8 \\
\hline
\end{tabular}

By contrast, polyamides $\mathbf{1 0 - 1 3}$ exhibited a decrease in both sensitivity and selectivity of the cation detection. This observation supported the important role played by the $\mathrm{NH}$ groups in the process of complexation. ${ }^{23,27,28}$

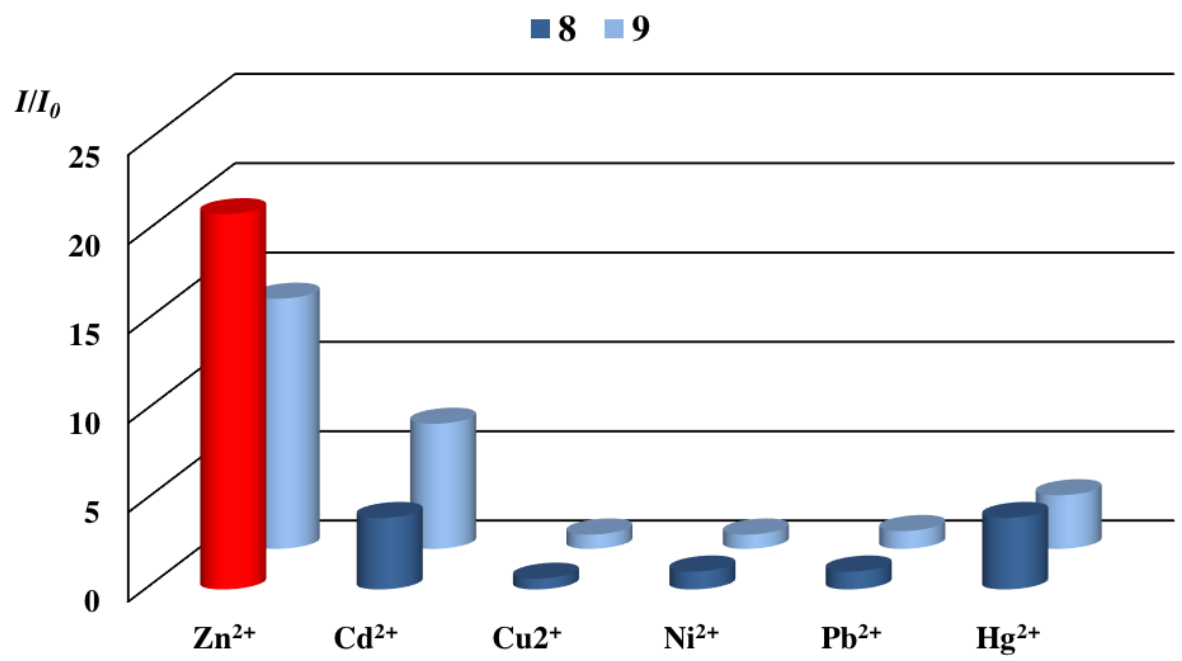

Figure 1. Changes in fluorescence intensity of polymers 8 and 9 in DMSO solutions in the presence of metal cations.

Addition of tetra-butylammonium salts (TBAX: $\mathrm{X}=\mathrm{F}, \mathrm{CN}, \mathrm{AcO}, \mathrm{NO}_{3}, \mathrm{HSO}_{4}$ ) to solutions of polymers 8-13 in DMSO leads to changes in absorption spectra (Fig. 2a). However, the appearance of a new intense absorption band in the region of 290-320 nm was observed exclusively for $\mathrm{F}^{-}, \mathrm{CN}^{-}$and, in particular, $\mathrm{AcO}^{-}$anions. According to preliminary DFT calculations, this process is associated with the substitution of chloride and the formation of much more stable (at $60-75 \mathrm{kcal} / \mathrm{mol}$ ) salt structures. Apparently, the corresponding redistribution of the electron density is the cause of the observed chromogenic "naked-eye" effect. ${ }^{29,30,31}$ 

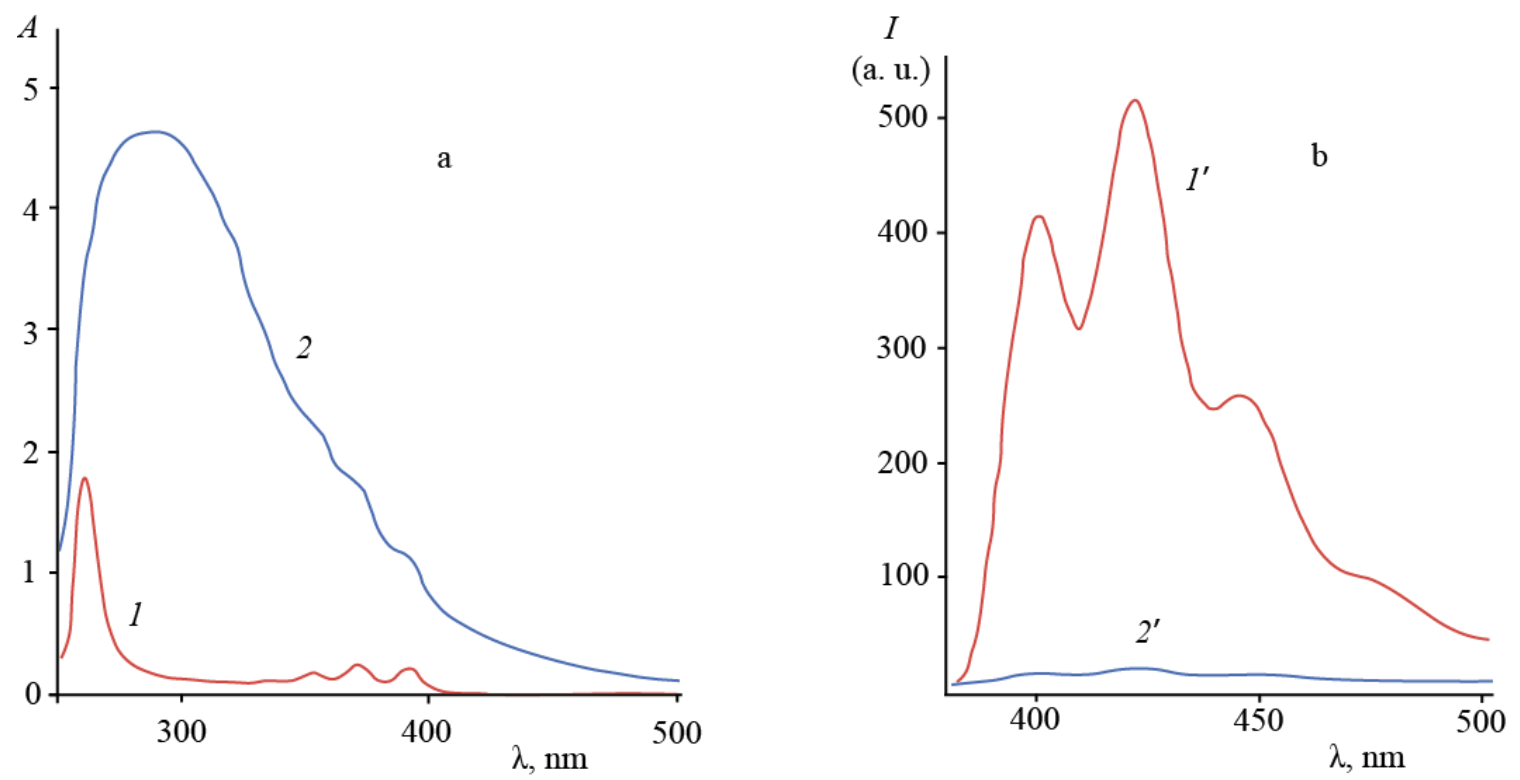

Figure 2. Absorption (a) and fluorescence (b) spectra of polymer 11 (DMSO, c 5.0 $\left.10^{-6} \mathrm{M}\right)$ before $\left(1,1^{\prime}\right)$ and after $\left(2,2^{\prime}\right)$ addition of $\mathrm{NBu}_{4}{ }^{+} \mathrm{AcO}^{-}\left(c 2.5 \cdot 10^{-5} \mathrm{M}\right)$.

Table 2. Relative change in fluorescence intensities of polymers 8-13 $\left(c 5.0 \cdot 10^{-6} \mathrm{M}\right)$ in DMSO upon addition of anions (in the form of TBAX salts) $\left(c 2.5 \cdot 10^{-5} \mathrm{M}\right)$

\begin{tabular}{cccccc}
\hline \multirow{2}{*}{ Polymer } & \multicolumn{5}{c}{ Relative change in fluorescence intensity, $\mathrm{I} / \mathrm{I}_{0}$} \\
\cline { 2 - 5 } & $\mathrm{F}^{-}$ & $\mathrm{CN}^{-}$ & $\mathrm{AcO}^{-}$ & $\mathrm{NO}_{3}{ }^{-}$ & $\mathrm{HSO}_{4}{ }^{-}$ \\
\hline $\mathbf{8}$ & 0.82 & 0.27 & 0.12 & 1.00 & 1.00 \\
$\mathbf{9}$ & 1.21 & 0.18 & 0.12 & 1.00 & 0.93 \\
$\mathbf{1 0}$ & 0.59 & 0.39 & 0.081 & 0.70 & 0.98 \\
$\mathbf{1 1}$ & 0.32 & 0.34 & 0.029 & 0.76 & 0.92 \\
12 & 0.76 & 0.49 & 0.063 & 1.00 & 0.90 \\
13 & 0.13 & 0.32 & 0.048 & 1.00 & 1.00 \\
\hline
\end{tabular}

Unlike cations, $\mathrm{F}^{-}, \mathrm{CN}^{-}, \mathrm{AcO}^{-}$and, to a lesser extent, some other anions cause only $\mathrm{CHEQ}$ effect (CHelationEnhanced fluorescence Quenching) (Fig. 2b, Table 2). ${ }^{3}$ Emission quenching is insufficiently suited for fluorimetric sensing, because it is unspecific and may be caused by several different external factors. ${ }^{22}$

\section{Conclusions}

Polymer fluorescent chemosensors based on poly(1-vinylimidazole) framework were synthesized. Aminoalkyl containing polymers exhibit chemosensor activity to $\mathrm{M}^{2+} d$-metal cations. Poly-(\{3-[2-(anthracen-9ylmethyl)amino]ethyl\}-1-vinylimidazolium chloride) selectively responds to the presence of zinc(II) cations by a 21-fold increase in relative fluorescence intensity of its DMSO solution. Amidoalkyl polymers are sensitive to $\mathrm{F}^{-}$ , $\mathrm{CN}^{-}$and $\mathrm{AcO}^{-}$anions. Poly-(\{3-[2-(anthracen-9-ylmethyl)(3,5-dichlorophenyl)amino]-2-oxoethyl\}-1-vinylimidazolium chloride) exhibits properties of a "naked-eye" chromogenic chemosensor for AcO- (change of the solution color from pale yellow to bright orange). 


\section{Experimental Section}

General. The IR spectra were recorded on a Varian Excalibur 3100 FT-IR instrument using the attenuated total internal reflection technique ( $\mathrm{ZnSe}$ crystal). The ${ }^{1} \mathrm{H}$ NMR spectra in DMSO- $d_{6}$ were recorded on a Varian Unity 300 spectrometer $(300 \mathrm{MHz})$, the signals were referenced with respect to the signals of residual protons of deutero-solvent (2.50 ppm). DMSO of the spectroscopic grade, $d$-metal perchlorates and tetrabutylammonium salts (Aldrich) were used to prepare solutions. Melting points were determined on a PTP (M) instrument. Elemental analysis was performed by classical procedure. ${ }^{32}$ Poly(1-vinylimidazole) was synthesized according to the literature. ${ }^{33}$

2-[(Anthracen-9-ylmethyl)amino]ethanol (1a). 1) Anthracene-9-carbaldehyde (20 mmol, $4.1 \mathrm{~g})$ was dissolved in toluene $(50 \mathrm{~mL})$, aminoethanol $(30 \mathrm{mmol}, 1.8 \mathrm{~mL})$ and $\mathrm{AcOH}(0.1 \mathrm{~mL})$ were added to the solution. The reaction mixture was refluxed for $6 \mathrm{~h}$, the solvent was removed on a rotary evaporator. The residue was cooled and recrystallized from $n$-BuOH. 2-[(Anthracen-9-ylmethylene)amino]ethan-1-ol (yellow fibrous solid) was filtered, washed on the filter with hot petroleum ether $(3 \times 10 \mathrm{~mL})$ and dried. Yield $86 \%(4.30 \mathrm{~g})$. The obtained imine was chromatographically pure and was used without further purification. 2) To a solution of 2[(anthracen-9-ylmethylene)amino]ethanol (15 mmol, $3.8 \mathrm{~g})$ in EtOH/DMF mixture (80:20) (50 mL) was added $\mathrm{NaBH}_{4}(50 \mathrm{mmol}, 1.9 \mathrm{~g})$ in small portions while stirring and heating to $40-50{ }^{\circ} \mathrm{C}$. The mixture was stirred for $1 \mathrm{~h}$ at $40-50{ }^{\circ} \mathrm{C}$, cooled to room temperature, and diluted with warm water $(100 \mathrm{~mL})$. The excess of $\mathrm{NaBH}_{4} \mathrm{Was}$ decomposed by $5 \% \mathrm{AcOH}$. An aqueous suspension was cooled to room temperature, the precipitate was filtered, washed on the filter with water $(3 \times 50 \mathrm{~mL})$, dried at $90-100{ }^{\circ} \mathrm{C}$ in a drying chamber for $4 \mathrm{~h}$ and recrystallized to give the title compound 1a. Yield $90 \%(3.4 \mathrm{~g})$, light yellow fibrous solid, $\mathrm{mp} 118-119{ }^{\circ} \mathrm{C}(n$ $\mathrm{BuOH})$. IR $\left(v, \mathrm{~cm}^{-1}\right): 3300,3243,1465,1365 .{ }^{1} \mathrm{H}$ NMR $\left(300 \mathrm{MHz}, \mathrm{DMSO}-d_{6}\right): \delta_{\mathrm{H}} 2.88\left(\mathrm{t}, 2 \mathrm{H}, J 5.4 \mathrm{~Hz}, \mathrm{NCH}_{2}\right), 3.57$ (t, $\left.2 \mathrm{H}, J 5.3 \mathrm{~Hz}, \mathrm{CH}_{2} \mathrm{O}\right), 4.29$ (br.s, $\left.1 \mathrm{H}, \mathrm{NH}\right), 4.65\left(\mathrm{~s}, 2 \mathrm{H}, \mathrm{CH}_{2} \mathrm{~N}\right), 7.40-7.56(\mathrm{~m}, 4 \mathrm{H}, \mathrm{Ar} \mathrm{H}), 8.01(\mathrm{~d}, 2 \mathrm{H}, J 8.1 \mathrm{~Hz}, \mathrm{Ar}$ $\mathrm{H}), 8.38(\mathrm{~d}, 2 \mathrm{H}, J 8.1 \mathrm{~Hz}, \mathrm{ArH}), 8.42(\mathrm{~s}, 1 \mathrm{H}, \mathrm{Ar} H)$. Anal. calcd. for $\mathrm{C}_{17} \mathrm{H}_{17} \mathrm{NO}: \mathrm{C}, 81.24 ; \mathrm{H}, 6.82 ; \mathrm{N}, 5.57$. Found: $\mathrm{C}$, 81.32; H, 6.90; N, 5.49\%.

3-[(Anthracen-9-ylmethyl)amino]propan-1-ol (1b) was synthesized at the same manner as 1 a from anthracene-9-carbaldehyde and 3-aminopropan-1-ol. Yield 72\% (2.9 g), pale green solid, mp $83-84{ }^{\circ} \mathrm{C}(\mathrm{PhH})$. IR $\left(v, \mathrm{~cm}^{-1}\right): 3315,3218,1474,1360 .{ }^{1} \mathrm{H}$ NMR $\left(300 \mathrm{MHz}, \mathrm{DMSO}-d_{6}\right): \delta_{\mathrm{H}} 1.78\left(\mathrm{q}, 2 \mathrm{H}, \mathrm{CCH}_{2} \mathrm{C}\right), 3.14(\mathrm{t}, 2 \mathrm{H}, J 5.7 \mathrm{~Hz}$, $\mathrm{NCH}_{2}$ ), $3.84\left(\mathrm{t}, 2 \mathrm{H}, J 5.7 \mathrm{~Hz}, \mathrm{CH}_{2} \mathrm{O}\right), 4.76\left(\mathrm{~s}, 2 \mathrm{H}, \mathrm{CH}_{2} \mathrm{~N}\right), 7.42-7.61(\mathrm{~m}, 4 \mathrm{H}, \mathrm{Ar} \mathrm{H}), 8.02(\mathrm{~d}, 2 \mathrm{H}, J 8.4 \mathrm{~Hz}, \mathrm{Ar} \mathrm{H}), 8.31$ (d, $2 \mathrm{H}, J 8.4 \mathrm{~Hz}, \mathrm{Ar} \mathrm{H}), 8.43$ (s, $1 \mathrm{H}, \mathrm{Ar} H$ ). Anal. calcd. for $\mathrm{C}_{18} \mathrm{H}_{19} \mathrm{NO}: \mathrm{C}, 81.47 ; \mathrm{H}, 7.22 ; \mathrm{N}, 5.28$. Found: $\mathrm{C}, 81.54$; $\mathrm{H}, 7.28 ; \mathrm{N}, 5.19 \%$.

$\boldsymbol{N}$-(Anthracen-9-ylmethyl)-1-phenylethanamine (1c) was synthesized at the same manner as $1 \mathrm{a}$ from anthracene-9-carbaldehyde and (S)-(-)-1-phenylethylamine. Yield 78\% (3.6 g), light yellow solid, $\mathrm{mp} 76-77{ }^{\circ} \mathrm{C}$ (PhH). IR (v, cm $\left.{ }^{-1}\right): 3310,1478,1375 .{ }^{1} \mathrm{H}$ NMR (300 MHz, DMSO-d 6 ): $\delta_{\mathrm{H}} 1.42\left(\mathrm{~d}, 3 \mathrm{H}, \mathrm{J} 7.4 \mathrm{~Hz}, \mathrm{CH}_{3}\right), 4.10(\mathrm{~d} . \mathrm{d}, 1 \mathrm{H}$, $J_{1} 4.8 \mathrm{~Hz}, J_{2} 14.8 \mathrm{~Hz}, \mathrm{CH}$ ), 4.53 (d.d, $2 \mathrm{H}, J_{1} 13.4 \mathrm{~Hz}, J_{2} 16.8 \mathrm{~Hz}, \mathrm{CH}_{2}$ ), 7.18-7.60 (m, 9H, Ar H), 7.90-8.20 (m, 5H, Ar $\mathrm{H}, \mathrm{NH}), 8.40(\mathrm{~s}, 1 \mathrm{H}, \mathrm{Ar} \mathrm{H})$. Anal. calcd. for $\mathrm{C}_{18} \mathrm{H}_{19} \mathrm{~N}$ : C, 88.71; H, 6.80; N, 4.49. Found: C, 88.62; H, 6.86; N, 4.52\%. $\mathbf{N}$-(Anthracen-9-ylmethyl)-3,5-dichloroaniline (1d) was synthesized at the same manner as 1 a from anthracene-9-carbaldehyde and 3,5-dichloroaniline. Yield 93\% (4.9 g), light yellow solid, mp 201-202 ${ }^{\circ} \mathrm{C}$ ( $n$ $\mathrm{BuOH})$. IR $\left(\mathrm{v}, \mathrm{cm}^{-1}\right): 3250,1600,1495,1400 .{ }^{1} \mathrm{H}$ NMR (300 MHz, DMSO-d $\left.)\right): \delta_{\mathrm{H}} 4.05$ (br.s, $\left.1 \mathrm{H}, \mathrm{NH}\right), 5.12(\mathrm{~d}, 2 \mathrm{H}, J$ 5.4 Hz, CH 2$), 7.15-7.40(m, 3 \mathrm{H}, \mathrm{Ar} \mathrm{H}), 7.46-7.62(\mathrm{~m}, 4 \mathrm{H}, \mathrm{ArH}), 8.04(\mathrm{~d}, 2 \mathrm{H}, J 8.4 \mathrm{~Hz}, \mathrm{Ar} \mathrm{H}), 8.42(\mathrm{~d}, 2 \mathrm{H}, J 8.2 \mathrm{~Hz}$, $\operatorname{Ar} H$ ), 8.58 (s, $1 \mathrm{H}, \operatorname{Ar} H$ ). Anal. calcd. for $\mathrm{C}_{21} \mathrm{H}_{15} \mathrm{Cl}_{2} \mathrm{~N}$ : C, 71.60; $\mathrm{H}, 4.29 ; \mathrm{Cl}, 20.13 ; \mathrm{N}, 3.98$. Found: $\mathrm{C}, 71.52 ; \mathrm{H}$, 4.36; $\mathrm{Cl}, 20.21 ; \mathrm{N}, 3.91 \%$. 
$\mathbf{N}$-(Anthracen-9-ylmethyl)-2-chloroethanaminium chloride (2). Compound 1 a $(10 \mathrm{mmol}, 2.5 \mathrm{~g})$ was dissolved in dry $\mathrm{CHCl}_{3}(100 \mathrm{~mL})$ while heating to $45-50{ }^{\circ} \mathrm{C}$, and a solution of fresh $\mathrm{SOCl}_{2}(12.5 \mathrm{mmol}, 0.9 \mathrm{~mL})$ in dry $\mathrm{CHCl}_{3}$ $(10 \mathrm{~mL})$ was added to the reaction mixture for 15-20 min with intensive stirring. The reaction mixture was then stirred for $30 \mathrm{~min}$, gradually heated and refluxed for $2 \mathrm{~h}$, and then evaporated to $10-15 \mathrm{~mL}$ on a rotary evaporator. The residue was cooled to $0{ }^{\circ} \mathrm{C}$, the precipitate was filtered, and washed on the filter by cooled $\mathrm{CHCl}_{3}(2 \times 5 \mathrm{~mL})$ and acetone $(2 \times 5 \mathrm{~mL})$. The resulting hydrochloride was recrystallized to afford the title compound 2. Yield 92\% (2.8 g), pale yellow solid, mp 222-223 ${ }^{\circ} \mathrm{C}(n-\mathrm{BuOH})$. 1365, 1017, 756. ${ }^{1} \mathrm{H}$ NMR (300 MHz, DMSO- $\left.d_{6}\right): \delta_{\mathrm{H}} 3.44-3.63\left(\mathrm{~m}, 2 \mathrm{H}, \mathrm{NCH}_{2}\right), 4.00\left(\mathrm{t}, 2 \mathrm{H}, J 6.2 \mathrm{~Hz}, \mathrm{CH}_{2} \mathrm{Cl}\right), 5.26$ (br.s, $2 \mathrm{H}, \mathrm{CH}_{2} \mathrm{~N}$ ), 7.50-7.73 (m, 4H, Ar H), $8.12(\mathrm{~d}, 2 \mathrm{H}, J 8.6 \mathrm{~Hz}, \mathrm{Ar} \mathrm{H}), 8.55(\mathrm{~d}, 2 \mathrm{H}, J 8.6 \mathrm{~Hz}, \mathrm{Ar} \mathrm{H}), 8.71(\mathrm{~s}, 1 \mathrm{H}, \mathrm{Ar}$ H), 9.85 (br.s, $2 \mathrm{H}, \mathrm{N}^{+} \mathrm{H}_{2}$ ). Anal. calcd. for $\mathrm{C}_{17} \mathrm{H}_{17} \mathrm{Cl}_{2} \mathrm{~N}$ : C, 66.68; $\mathrm{H}, 5.60 ; \mathrm{Cl}, 23.15 ; \mathrm{N}, 4.57$. Found: $\mathrm{C}, 66.75 ; \mathrm{H}$, 5.55; $\mathrm{Cl}, 23.08 ; \mathrm{N}, 4.62 \%$.

$\mathbf{N}$-(Anthracen-9-ylmethyl)-3-chloropropan-1-aminium chloride (3) was synthesized at the same manner as compound 2. Yield $82 \%$ (2.6 g), light yellow solid, $\mathrm{mp} 200-201{ }^{\circ} \mathrm{C}(n-\mathrm{BuOH})$. IR $\left(\mathrm{v}, \mathrm{cm}^{-1}\right): 3300,3245,1465$, 1400, 1005, 761. ${ }^{1} \mathrm{H}$ NMR (300 MHz, DMSO- $\left.d_{6}\right): \delta_{\mathrm{H}} 1.90-2.05\left(\mathrm{~m}, 2 \mathrm{H}, \mathrm{CCH}_{2} \mathrm{C}\right), 3.44\left(\mathrm{t}, 2 \mathrm{H}, J 6.2 \mathrm{~Hz}, \mathrm{CH}_{2} \mathrm{Cl}\right), 4.14-$ $4.25\left(\mathrm{~m}, 2 \mathrm{H}, \mathrm{NCH}_{2}\right), 5.18\left(\mathrm{~s}, 2 \mathrm{H}, \mathrm{CH}_{2} \mathrm{~N}\right), 7.38-7.55(\mathrm{~m}, 4 \mathrm{H}, \mathrm{Ar} \mathrm{H}), 8.07(\mathrm{~d}, 2 \mathrm{H}, J 8.6 \mathrm{~Hz}, \mathrm{Ar} \mathrm{H}), 8.42(\mathrm{~d}, 2 \mathrm{H}, J 8.6 \mathrm{~Hz}$, $\operatorname{Ar~H}), 8.57$ (s, $1 \mathrm{H}, \mathrm{Ar} \mathrm{H}$ ), 10.11 (br.s, $2 \mathrm{H}, \mathrm{N}^{+} \mathrm{H}_{2}$ ). Anal. calcd. for $\mathrm{C}_{18} \mathrm{H}_{19} \mathrm{Cl}_{2} \mathrm{~N}: \mathrm{C}, 67.51 ; \mathrm{H}, 5.98 ; \mathrm{Cl}, 22.14 ; \mathrm{N}, 4.37$. Found: $\mathrm{C}, 67.61 ; \mathrm{H}, 6.07 ; \mathrm{Cl}, 22.06 ; \mathrm{N}, 4.46 \%$.

$\boldsymbol{N}$-(Anthracen-9-ylmethyl)-2-chloro- $\boldsymbol{N}$-(1-phenylethyl)acetamide (4). Amine 1c (10 mmol, $3.1 \mathrm{~g})$ was dissolved in dry toluene $(50 \mathrm{~mL})$ and a solution of chloroacetyl chloride $(12.5 \mathrm{mmol}, 1.0 \mathrm{~mL})$ in dry pyridine $(20 \mathrm{mmol}$, $1.6 \mathrm{~mL}$ ) was added with intense stirring. The reaction mixture was refluxed for $6 \mathrm{~h}$, cooled to $5-10{ }^{\circ} \mathrm{C}$ and the precipitate of pyridine hydrochloride was filtered off. The filtrate was washed with water $(2 \times 20 \mathrm{~mL})$ in a separating funnel to remove the residue of pyridine hydrochloride. The resulting toluene solution was dried $\left(\mathrm{Na}_{2} \mathrm{SO}_{4}\right)$ for $1 \mathrm{~h}$. Then $\mathrm{Na}_{2} \mathrm{SO}_{4}$ was filtered off, the solvent was removed on a rotary evaporator, the residue was recrystallized to afford a yellow precipitate which was filtered, washed with petroleum ether $(3 \times 10 \mathrm{~mL})$ and dried to afford the title compound 4. Yield 75\% $(2.9 \mathrm{~g}), \mathrm{mp} 64-65^{\circ} \mathrm{C}\left(n\right.$-hexane/PhH, 75:25). IR $\left(\mathrm{V}, \mathrm{cm}^{-1}\right)$ : 1625, 1500, 1440, 1022. ${ }^{1} \mathrm{H}$ NMR (300 MHz, DMSO-d 6 ): $\delta_{\mathrm{H}} 1.45$ (d, $\left.3 \mathrm{H}, J 7.0 \mathrm{~Hz}, \mathrm{CH}_{3}\right), 3.80\left(\mathrm{~s}, 2 \mathrm{H}, \mathrm{CH}_{2} \mathrm{Cl}\right), 4.02$ (d.d, $\left.1 \mathrm{H}, J_{1} 5.0 \mathrm{~Hz}, J_{2} 16.0 \mathrm{~Hz}, \mathrm{CH}\right), 5.21\left(\mathrm{~d}, 2 \mathrm{H}, \mathrm{J} 7.2 \mathrm{~Hz}, \mathrm{NCH}_{2}\right), 7.18-7.60(\mathrm{~m}, 9 \mathrm{H}, \mathrm{Ar} \mathrm{H}), 7.97-8.20(\mathrm{~m}, 3 \mathrm{H}, \mathrm{Ar} \mathrm{H}$ ), $8.33(\mathrm{~d}, 2 \mathrm{H}, \mathrm{J} 8.8 \mathrm{~Hz}, \mathrm{Ar} \mathrm{H})$. Anal. calcd. for $\mathrm{C}_{25} \mathrm{H}_{22} \mathrm{ClNO}$ : C, 77.41; $\mathrm{H}, 5.72 ; \mathrm{Cl}, 9.14 ; \mathrm{N}, 3.61$. Found: $\mathrm{C}, 77.48 ; \mathrm{H}$, 5.67; $\mathrm{Cl}, 9.20 ; \mathrm{N}, 3.54 \%$.

$\mathbf{N}$-(Anthracen-9-ylmethyl)-2-chloro- $\mathbf{N}$-(3,5-dichlorophenyl)acetamide (5) was synthesized in the same manner as $\mathbf{4}$ from amine $\mathbf{1 d}$ and chloroacetyl chloride. Yield $84 \%$ (3.6 g), yellow solid, mp $177-178{ }^{\circ} \mathrm{C}$ (THF). IR $\left(v, \mathrm{~cm}^{-1}\right): 1620,1495,1430,1240,1005,759 .{ }^{1} \mathrm{H}$ NMR $\left(300 \mathrm{MHz}, \mathrm{DMSO}-d_{6}\right): \delta_{\mathrm{H}} 3.97\left(\mathrm{~s}, 2 \mathrm{H}, \mathrm{CH}_{2} \mathrm{Cl}\right), 6.05(\mathrm{~s}, 2 \mathrm{H}$, $\left.\mathrm{CH}_{2} \mathrm{~N}\right)$, 7.11-7.41 (m, 3H, Ar H), 7.46-7.62 (m, 4H, Ar H), $8.03(\mathrm{~d}, 2 \mathrm{H}, J 8.8 \mathrm{~Hz}, \mathrm{Ar} \mathrm{H}), 8.46(\mathrm{~d}, 2 \mathrm{H}, J 8.8 \mathrm{~Hz}, \mathrm{Ar} \mathrm{H})$, 8.60 (s, $1 \mathrm{H}, \mathrm{Ar} \mathrm{H}$ ). Anal. calcd. for $\mathrm{C}_{24} \mathrm{H}_{18} \mathrm{Cl}_{3} \mathrm{NO}: \mathrm{C}, 65.10 ; \mathrm{H}, 4.10 ; \mathrm{Cl}, 24.02 ; \mathrm{N}, 3.16$. Found: $\mathrm{C}, 65.18 ; \mathrm{H}, 4.18 ; \mathrm{Cl}$, 23.94; N, 3.04\%.

$\boldsymbol{N}$-(Anthracen-9-ylmethyl)-3-chloro- $\boldsymbol{N}$-(1-phenylethyl)propanamide (6) was synthesized at the same manner as 4 from amine $1 \mathrm{c}$ and 3-chloropropionyl chloride. Yield $70 \%(2.8 \mathrm{~g})$, light yellow solid, $\mathrm{mp} 56-57{ }^{\circ} \mathrm{C}(n-$ hexane/PhH, 80:20). IR $\left(v, \mathrm{~cm}^{-1}\right): 1630,1485,1405,1009 .{ }^{1} \mathrm{H}$ NMR $\left(300 \mathrm{MHz}, \mathrm{DMSO}-d_{6}\right): \delta_{\mathrm{H}} 1.42(\mathrm{~d}, 3 \mathrm{H}, J 6.8$ $\left.\mathrm{Hz}, \mathrm{CH}_{3}\right), 2.91\left(\mathrm{~d}, 2 \mathrm{H}, J 6.8 \mathrm{~Hz}, \mathrm{CCH}_{2} \mathrm{C}\right), 3.42\left(\mathrm{~d}, 2 \mathrm{H}, J 6.8 \mathrm{~Hz}, \mathrm{CH}_{2} \mathrm{Cl}\right), 3.94$ (d.d, $\left.1 \mathrm{H}, J_{1} 5.0 \mathrm{~Hz}, J_{2} 15.0 \mathrm{~Hz}, \mathrm{CH}\right), 5.04$ (d, $\left.2 \mathrm{H}, J 6.8 \mathrm{~Hz}, \mathrm{NCH}_{2}\right), 7.14-7.55(\mathrm{~m}, 9 \mathrm{H}, \mathrm{Ar} \mathrm{H}), 7.92-8.17(\mathrm{~m}, 3 \mathrm{H}, \mathrm{Ar} \mathrm{H}), 8.40(\mathrm{~d}, 2 \mathrm{H}, J 8.6 \mathrm{~Hz}, \mathrm{Ar} \mathrm{H})$. Anal. calcd. for $\mathrm{C}_{25} \mathrm{H}_{16} \mathrm{Cl}_{3} \mathrm{NO}$ : C, 64.43; $\mathrm{H}, 3.76 ; \mathrm{Cl}, 24.81 ; \mathrm{N}, 3.27$. Found: $\mathrm{C}, 64.50 ; \mathrm{H}, 3.68 ; \mathrm{Cl}, 24.90 ; \mathrm{N}, 3.20 \%$.

$\boldsymbol{N}$-(Anthracen-9-ylmethyl)-3-chloro- $\mathbf{N}$-(3,5-dichlorophenyl)propanamide (7) was synthesized in the same manner as 4 from amine 1d and 3-chloropropionyl chloride. Yield 80\% (3.5 g), light green-yellow solid, mp 172$173{ }^{\circ} \mathrm{C}(n-\mathrm{BuOH})$. IR $\left(v, \mathrm{~cm}^{-1}\right): 1615,1500,1430,1235,1018,764 .{ }^{1} \mathrm{H}$ NMR $\left(300 \mathrm{MHz}, \mathrm{DMSO}-d_{6}\right): \delta_{\mathrm{H}} 2.96(\mathrm{~d}, 2 \mathrm{H}$, 
J $\left.7.0 \mathrm{~Hz}, \mathrm{CCH}_{2} \mathrm{C}\right), 3.55\left(\mathrm{~d}, 2 \mathrm{H}, J 7.0 \mathrm{~Hz}, \mathrm{CH}_{2} \mathrm{Cl}\right), 5.88\left(\mathrm{~s}, 2 \mathrm{H}, \mathrm{CH}_{2} \mathrm{~N}\right), 7.16-7.40(\mathrm{~m}, 3 \mathrm{H}, \mathrm{Ar} \mathrm{H}), 7.50-7.65(\mathrm{~m}, 4 \mathrm{H}, \mathrm{Ar}$ $\mathrm{H}), 8.05(\mathrm{~d}, 2 \mathrm{H}, J 8.6 \mathrm{~Hz}, \mathrm{Ar} \mathrm{H}), 8.42(\mathrm{~d}, 2 \mathrm{H}, J 8.6 \mathrm{~Hz}, \mathrm{Ar} \mathrm{H}), 8.52$ (s, $1 \mathrm{H}, \mathrm{Ar} \mathrm{H})$. Anal. calcd. for $\mathrm{C}_{24} \mathrm{H}_{18} \mathrm{Cl}_{3} \mathrm{NO} \mathrm{C}$, 65.10; H, 4.10; Cl, 24.02; N, 3.16.Found: C, 65.18; H, 4.18; Cl, 23.94; N, 3.04\%.

Poly-(\{3-[2-(anthracen-9-ylmethyl)amino]ethyl\}-1-vinylimidazolium chloride) (8). 1) $N$-(Anthracen-9-ylmethyl)-2-chloroethanaminium chloride (2) $(6.5 \mathrm{mmol}, 2.0 \mathrm{~g})$ was treated with stirring with $10 \%$ aqueous $\mathrm{Na}_{2} \mathrm{CO}_{3}$ solution $(50 \mathrm{~mL}$ ) in a $100 \mathrm{~mL}$ glass beaker. After 30 min the resulting suspension was transferred into separating funnel together with suspension residues washed out of the beaker with chloroform $(2 \times 10 \mathrm{~mL}) . N$ (Anthracen-9-ylmethyl)-2-chloroethanamine was extracted with $\mathrm{CHCl}_{3}(3 \times 50 \mathrm{~mL})$, washed with water $(2 \times 100$ $\mathrm{mL}$ ) and dried $\left(\mathrm{Na}_{2} \mathrm{SO}_{4}\right)$ for $2 \mathrm{~h}$. Chloroform solution was evaporated on a rotary evaporator to $10-15 \mathrm{~mL}$. 2) Poly(1-vinylimidazole) $(5.3 \mathrm{mmol}$ in terms of monomer, $0.50 \mathrm{~g}$ ) was dissolved under heating in $100 \mathrm{~mL}$ of $n$ $\mathrm{BuOH} / \mathrm{EtOH}$ (80:20) mixture and a solution of $\mathrm{N}$-(anthracen-9-ylmethyl)-2-chloroethanamine in $\mathrm{CHCl}_{3}$, obtained after evaporation, was carefully added. The resulting mixture was slowly evaporated to $20-25 \mathrm{~mL}$ and then refluxed for 20-22 $\mathrm{h}$. The solvent was evaporated to $1 / 4$ volume on a rotary evaporator; the obtained solution was cooled, and diluted with acetone $(100 \mathrm{~mL})$. The reaction mixture was thoroughly stirred for $1.5-2$ $h$, the precipitate was filtered, and washed on the filter with cold acetone $(3 \times 10 \mathrm{~mL})$. The residue was thoroughly shredded and dried in vacuo at $75-80{ }^{\circ} \mathrm{C}$ in a drying chamber to give the title polymer 8 . Yield $72 \%$ $(1.50 \mathrm{~g}), \mathrm{mp}>185^{\circ} \mathrm{C}$ (dec.). IR (, $\left.\mathrm{cm}^{-1}\right): 3288,3262,1612,1502,1091 .{ }^{1} \mathrm{H}$ NMR $\left(300 \mathrm{MHz}\right.$, DMSO- $\left.d_{6}\right): \delta_{\mathrm{H}} 1.50-$ $2.70\left[\mathrm{~m},(3 \mathrm{H})_{\mathrm{n}},\left(\mathrm{CHCH}_{2}\right)_{n}\right], 3.60-4.80\left[\mathrm{~m},(4 \mathrm{H})_{\mathrm{n}}, 2\left(\mathrm{CH}_{2}\right)_{\mathrm{n}}\right], 5.60-6.35\left[\mathrm{~m},(2 \mathrm{H})_{\mathrm{n}},\left(\mathrm{CH}_{2}\right)_{\mathrm{n}}\right], 6.80-9.30\left[\mathrm{~m},(12 \mathrm{H})_{\mathrm{n}},(\mathrm{Ar}\right.$ $\mathrm{H})_{n}$ ]. Anal. calcd for $\left(\mathrm{C}_{22} \mathrm{H}_{22} \mathrm{ClN}_{3}\right)_{n}: \mathrm{C}, 72.62 ; \mathrm{H}, 6.09 ; \mathrm{Cl}, 9,74 ; \mathrm{N}, 11.55$. Found: $\mathrm{C}, 72.76 ; \mathrm{H}, 5.94 ; \mathrm{Cl}, 9,87 ; \mathrm{N}$, $11.43 \%$.

Poly-(\{3-[3-(anthracen-9-ylmethyl)amino]propyl\}-1-vinylimidazolium chloride) (9) was synthesized at the same manner as 8 using chloride 3 and poly-(1-vinylimidazole). Yield $65 \%(1.4 \mathrm{~g}), \mathrm{mp}>155^{\circ} \mathrm{C}\left(\mathrm{dec}\right.$.). IR $\left(\mathrm{v}, \mathrm{cm}^{-}\right.$ $\left.{ }^{1}\right): 1671,1504,1408,1252,1003 .{ }^{1} \mathrm{H}$ NMR $\left(300 \mathrm{MHz}, \mathrm{DMSO}-d_{6}\right): \delta_{\mathrm{H}} 1.60-6.40\left[\mathrm{~m},(12 \mathrm{H})_{\mathrm{n}},\left(\mathrm{CH}_{2} \mathrm{CH}_{2} \mathrm{CH}_{2}\right)_{\mathrm{n}}\right.$, $\left.\left(\mathrm{CHCH}_{2}\right)_{n},\left(\mathrm{CH}_{2}\right)_{n},(\mathrm{NH})_{n}\right], 6.65-9.35\left[\mathrm{~m},(12 \mathrm{H})_{n},(\mathrm{Ar} \mathrm{H})_{n}\right]$. Anal. calcd for $\left(\mathrm{C}_{23} \mathrm{H}_{24} \mathrm{ClN}_{3}\right)_{n}$ : C, 73.10; $\mathrm{H}, 6.40 ; \mathrm{Cl}, 9,38$; $\mathrm{N}, 11.12$. Found: $\mathrm{C}, 73.22 ; \mathrm{H}, 6.30 ; \mathrm{Cl}, 9,50 ; \mathrm{N}, 10.98 \%$.

Poly-(\{3-[2-(anthracen-9-ylmethyl)(1-phenylethyl)amino]-2-oxoethyl\}-1-vinylimidazolium chloride) (10). Poly-(1-vinylimidazole) $(5.3 \mathrm{mmol}$ in terms of the monomer, $0.50 \mathrm{~g}$ ) was dissolved by heating in $n$ - $\mathrm{BuOH} / \mathrm{EtOH}$ $(80: 20)$ mixture $(150 \mathrm{~mL})$, acetamide $4(6.4 \mathrm{mmol}, 2.50 \mathrm{~g})$ was added, and the reaction mixture was refluxed for $12 \mathrm{~h}$ (after 6-7 $\mathrm{h}$ the formation of solution was observed). The solvent was evaporated on a rotary evaporator to $25-30 \mathrm{~mL}$, the obtained solution was cooled and diluted with acetone $(100 \mathrm{~mL})$. The reaction mixture was thoroughly stirred for $1 \mathrm{~h}$, the precipitate was filtered, and washed on the filter with cold acetone $(3 \times 10 \mathrm{~mL})$. The residue was thoroughly shredded and dried in vacuo at $65-70{ }^{\circ} \mathrm{C}$ in a drying chamber. Yield $75 \%$ (1.90 g), $\mathrm{mp}>130{ }^{\circ} \mathrm{C}$ (dec.). IR $\left(\mathrm{v}, \mathrm{cm}^{-1}\right): 1651,1479,1423,1265,1010 .{ }^{1} \mathrm{H}$ NMR $\left(300 \mathrm{MHz}, \mathrm{DMSO}-d_{6}\right): \delta_{\mathrm{H}}$ 1.70-3.25 [m, $\left.(6 \mathrm{H})_{n},\left(\mathrm{CH}_{3}\right)_{n},\left(\mathrm{CHCH}_{2}\right) \mathrm{n}\right], 4.30-5.20\left[\mathrm{~m},(3 \mathrm{H})_{\mathrm{n}},(\mathrm{CH})_{\mathrm{n}},\left(\mathrm{CH}_{2}\right) \mathrm{n}\right], 5.60-6.20\left[\mathrm{~m},(2 \mathrm{H})_{\mathrm{n}},\left(\mathrm{CH}_{2}\right) \mathrm{n}\right], 6.80-$ $9.45\left[\mathrm{~m},(17 \mathrm{H})_{n},(\mathrm{Ar} \mathrm{H})_{n}\right]$. Anal. calcd for $\left(\mathrm{C}_{30} \mathrm{H}_{28} \mathrm{ClN}_{3} \mathrm{O}\right)_{n}$ : C, 74.75; $\mathrm{H}, 5.86 ; \mathrm{Cl}, 7.35 ; \mathrm{N}, 8.72$. Found: $\mathrm{C}, 74.91 ; \mathrm{H}$, $5.77 ; \mathrm{Cl}, 7.49 ; \mathrm{N}, 8.62 \%$.

Poly-(\{3-[2-(anthracen-9-ylmethyl)(3,5-dichlorophenyl)amino]-2-oxoethyl\}-1-vinylimidazolium chloride) (11) was synthesized at the same manner as $\mathbf{1 0}$ using acetamide $\mathbf{5}$ and poly-(1-vinylimidazole). Yield $74 \%$ (x.y1.9 g), $\mathrm{mp}>175{ }^{\circ} \mathrm{C}$ (dec.). IR $\left(v, \mathrm{~cm}^{-1}\right): 1645,1500,1411,1244,1087 .{ }^{1} \mathrm{H}$ NMR $\left(300 \mathrm{MHz}, \mathrm{DMSO}-d_{6}\right): \delta_{\mathrm{H}} 2.25-3.05[\mathrm{~m}$, $\left.(3 \mathrm{H})_{n},\left(\mathrm{CHCH}_{2}\right)_{n}\right], 4.45-5.30\left[\mathrm{~m},(2 \mathrm{H})_{n},\left(\mathrm{CH}_{2}\right)_{n}\right], 5.65-6.30\left[\mathrm{~m},(2 \mathrm{H})_{n},\left(\mathrm{CH}_{2}\right)_{n}\right], 6.90-9.40\left[\mathrm{~m},(15 \mathrm{H})_{n},(\mathrm{Ar} \mathrm{H})_{n}\right]$. Anal. calcd for $\left(\mathrm{C}_{28} \mathrm{H}_{22} \mathrm{Cl}_{3} \mathrm{~N}_{3} \mathrm{O}\right)_{n}$ : C, 64.32; H, 4.24; Cl, 20.34; N, 8.04. Found: C, 64.45; H, 4.15; $\mathrm{Cl}, 20.45 ; \mathrm{N}, 7.92 \%$.

Poly-(\{3-[3-(anthracen-9-ylmethyl)(1-phenylethyl)amino]-3-oxopropyl\}-1-vinylimidazolium chloride) (12) was synthesized at the same manner as $\mathbf{1 0}$ from propanamide 6 and poly(1-vinylimidazole). Yield $77 \%$ (2.0 g), $\mathrm{mp}>165{ }^{\circ} \mathrm{C}$ (dec.). IR $\left(\mathrm{v}, \mathrm{cm}^{-1}\right): 1652,1488,1430,1232,1007 .{ }^{1} \mathrm{H}$ NMR (300 MHz, DMSO-d 6 ): $\delta_{\mathrm{H}} 1.85-3.50[\mathrm{~m}$, 
$\left.(8 \mathrm{H})_{n},\left(\mathrm{CH}_{3}\right)_{n},\left(\mathrm{CH}_{2}\right)_{n},\left(\mathrm{CHCH}_{2}\right)_{n}\right], 4.00-5.35\left[\mathrm{~m},(3 \mathrm{H})_{n},(\mathrm{CH})_{n},\left(\mathrm{CH}_{2}\right)_{n}\right], 5.80-6.30\left[\mathrm{~m},(2 \mathrm{H})_{n},\left(\mathrm{CH}_{2}\right)_{n}\right], 6.85-9.60[\mathrm{~m}$, $(17 \mathrm{H})_{n},(\mathrm{Ar} \mathrm{H})_{n}$ ]. Anal. calcd for $\left.\mathrm{C}_{31} \mathrm{H}_{30} \mathrm{ClN}_{3} \mathrm{O}\right)_{n}$ : C, 75.06; $\mathrm{H}, 6.10 ; \mathrm{Cl}, 7.15 ; \mathrm{N}, 8.47$. Found: $\mathrm{C}, 74.91 ; \mathrm{H}, 5.77 ; \mathrm{Cl}$, 7.49; N, 8.62\%.

Poly-(\{3-[3-(anthracen-9-ylmethyl)(3,5-dichlorophenyl)amino]-2-oxopropyl\}-1-vinylimidazolium chloride) (13) was synthesized at the same manner as $\mathbf{1 0}$ from propanamide 7 and poly(1-vinylimidazole). Yield $92 \%$ (2.4 g), $\mathrm{mp}>180{ }^{\circ} \mathrm{C}$ (dec.). IR (v, cm ${ }^{-1}$ ): 1660, 1495, 1415, 1255, 1078. ${ }^{1} \mathrm{H}$ NMR (300 MHz, DMSO- $\left.d_{6}\right): \delta_{\mathrm{H}} 1.75-2.80$ $\left[\mathrm{m},(3 \mathrm{H})_{n},\left(\mathrm{CHCH}_{2}\right)_{\mathrm{n}}\right], 3.15-3.40\left[\mathrm{~m},(2 \mathrm{H})_{\mathrm{n}},\left(\mathrm{CH}_{2}\right)_{\mathrm{n}}\right], 4.30-4.75\left[\mathrm{~m},(2 \mathrm{H})_{\mathrm{n}},\left(\mathrm{CH}_{2}\right)_{\mathrm{n}}\right], 5.65-6.10\left[\mathrm{~m},(2 \mathrm{H})_{\mathrm{n}},\left(\mathrm{CH}_{2}\right)_{\mathrm{n}}\right], 6.80-$ $10.00\left[\mathrm{~m},(15 \mathrm{H})_{n},(\mathrm{Ar} \mathrm{H})_{n}\right]$. Anal. calcd for $\left(\mathrm{C}_{29} \mathrm{H}_{24} \mathrm{Cl}_{3} \mathrm{~N}_{3} \mathrm{O}\right)_{n}$ : C, 64.88; $\mathrm{H}, 4.51 ; \mathrm{Cl}, 19.81 ; \mathrm{N}, 7.83$. Found: C, 65.01; $\mathrm{H}, 4.40 ; \mathrm{Cl}, 19.94 ; \mathrm{N}, 7.71 \%$.

\section{Acknowledgements}

This research was supported by the Russian Foundation for Basic Research (No. 16-03-00102). Yu. Revinskii and A. Dubonosov worked in the framework of the State Assignment for SSC RAS (No. 01201354239).

\section{References}

1. Wu, D.; Sedgwick, A. C.; Gunnlaugsson, T.; Akkaya, E. U.; Yoon, J.; James, T. D. Chem. Soc. Rev. 2017, 46, 7105.

http://dx.doi.org/10.1039/C7CSO0240H

2. Gale, P. A.; Caltagirone, C. Coord. Chem. Rev. 2018, 354, 2.

http://dx.doi.org/10.1016/i.ccr.2017.05.003

3. Chemosensors: Principles, Strategies, and Applications; Wang, B.; Anslyn, E. V. Eds.; Wiley, Hoboken, NJ, 2011.

4. Dubonosov, A. D.; Bren, V. A.; Minkin, V. I. Tautomerism: Concepts and Applications in Science and Technology; Antonov, L. Eds.; Wiley-VCH: Weinheim, 2016; pp 229-252.

5. Formica, M.; Fusi, V.; Giorgi, L.; Micheloni, M. Coord. Chem. Rev. 2012, 256, 170. https://doi.org/10.1016/j.ccr.2011.09.010

6. Daly, B.; Ling, J.; de Silva, A. P. Chem. Soc. Rev. 2015, 44, 4203. https://doi.org/10.1039/C4CS00334A

7. Sharma, H.; Kaur, N.; Singh, A.; Kuwar, A.; Singh, N. J. Mater. Chem. C 2016, 4, 5154. https://doi.org/10.1039/C6TC00605A

8. Kaur, N.; Kumar, S. Tetrahedron 2011, 67, 9233. https://doi.org/10.1016/i.tet.2011.09.003

9. Carter, K. P., Young, A. M., Palmer, A. E. Chem. Rev. 2014, 114, 4564. https://doi.org/10.1021/cr400546e

10. Yu, L.; Wang, S.; Huang, K.; Liu, Z.; Gao, F.; Zeng, W. Tetrahedron 2015, 71, 4679. https://doi.org/10.1016/j.tet.2015.04.115

11. Yang, Y.; Zhao, Q.; Feng, W.; Li, F. Chem. Rev. 2013, 113, 192. https://doi.org/10.1021/cr2004103

12. Skoda-Földes, R. Molecules 2014, 19, 8840. https://doi.org/10.3390/molecules19078840 
13. Nikitina, O.; Shleev, S.; Gayda, G.; Demkiv, O.; Gonchar, M.; Gorton, L.; Csöregi, E.; Nistor, M. Sens. Actuators, B 2007, 125, 1.

https://doi.org/10.1016/j.snb.2007.01.025

14. Sakaia, R.; Satoh, T.; Kakuchi, T. Polym. Rev. 2017, 57, 159.

https://doi.org/10.1080/15583724.2016.1144613

15. Chen, Z.; Lu, C. Sens. Lett. 2005, 3, 274.

https://doi.org/10.1166/sl.2005.045

16. Kuba, A. G.; Smolin, Y. Y.; Soroush, M.; Lau, K. K. S. Chem. Eng. Sci. 2016, 154, 136. https://doi.org/10.1016/i.ces.2016.05.007

17. García, J. M.; García, F. C.; Serna, F.; de la Peña, J. L. Polym. Rev. 2011, 51, 341. https://doi.org/10.1080/15583724.2011.616084

18. Ruiz, J. A. R.; Sanjuán, A. M.; Vallejos, S.; García, F. C.; García, J. M. Chemosensors 2018, 6, 12. https://doi.org/10.3390/chemosensors6020012

19. Attia, M. S.; Abdel-Mottaleb, M. S. A. Handbook of Polymers for Pharmaceutical Technologies: Processing and Applications; Kumar, T.V.; Thakur, M. K. Eds.; Wiley-Scrivener, Hoboken, NJ, 2015.

20. Guan, W.; Zhou, W.; Lu, J.; Lu, C. Chem. Soc. Rev. 2015, 44, 6981. http://dx.doi.org/10.1039/C5CS00246J

21. Kim, H. N.; Guo, Z.; Zhu, W.; Yoon, J.; Tian, H. Chem. Soc. Rev. 2011, 40, 79. http://dx.doi.org/10.1039/COCSO0058B

22. Granzhan, A.; Ihmels, H.; Tian, M. Arkivoc 2015, (vi), 494. http://dx.doi.org/10.3998/ark.5550190.p009.339

23. Tolpygin, I. E.; Bren, V. A.; Dubonosov, A. D.; Minkin, V. I.; Rybalkin, V. P. Russ. J. Org. Chem. 2003, 39, 1364.

https://doi.org/10.1023/B:RUJO.0000010232.50792.88

24. Tolpygin, I. E.; Rybalkin, V. P.; Shepelenko, E. N.; Popova, L. L.; Revinskii, Yu. V.; Tsukanov, A. V.; Dmitrieva, O. I.; Dubonosov, A. D.; Bren, V. A.; Minkin, V. I. Russ. J. Org. Chem. 2008, 44, 557. https://doi.org/10.1134/S1070428008040155

25. Wu J.; Liu W.; Ge J.; Zhang H.; Wang P. Chem. Soc. Rev. 2011, 40, 3483.

https://doi.org/10.1039/COCS00224K

26. Liu, Z.; He, W.; Guo, Z. Chem. Soc. Rev. 2013, 42, 1568.

https://doi.org/10.1039/C2CS35363F

27. Gribanova, T. N; Dubonosov, A. D.; Tolpygin, I. E.; Rybalkin, V. P.; Bren, V. A.; Minyaev, R. M.; Minkin, V. I. Russ. J. Org. Chem. 2005, 41, 1175. https://doi.org/10.1007/s11178-005-0311-y

28. Kaur, N.; Kaur, G.; Fegade, U. A.; Singh, A.; Sahoo, S. K.; Kuwar, A. S.; Singh, N. Trends Analyt. Chem. 2017, 95, 86.

https://doi.org/10.1016/j.trac.2017.08.003

29. Martınez-Manez, R.; Sancenon, F. Chem. Rev. 2003, 103, 4419.

https://doi.org/10.1021/cr010421e

30. Zhou, Y.; Zhang, J. F.; Yoon, J. Chem. Rev. 2014, 114, 5511.

https://10.1021/cr400352m

31. Kumar, R.; Sandhu, S.; Singh, P.; Kumar, S. Chem. Rec. 2017, 17, 1.

https://doi.org/10.1002/tcr.201600108 
32. Gel'man, N. E.; Terent'eva, E. A.; Shanina, T. M. Methods of Quantitative Organic Elemental Microanalysis [in Russian]; Gel'man, N. E., Ed.; Khimiya: Moscow, 1987.

33. Talu, M.; Demiroğlu, E. U.; Yurdakul, Ş.; Badoğlu, S. Spectrochim. Acta A 2015, 134, 267. https://doi.org/10.1016/i.saa.2014.06.101 